\title{
Resonant Higgs pair production as a probe of stop at the LHC
}

\author{
Guang Hua Duan, ${ }^{c, d}$ Lei $\mathbf{W} \mathbf{u}^{a, b}$ and Rui Zheng ${ }^{e}$ \\ ${ }^{a}$ Department of Physics and Institute of Theoretical Physics, Nanjing Normal University, \\ Nanjing, Jiangsu, 210023 China \\ ${ }^{b}$ ARC Centre of Excellence for Particle Physics at the Terascale, School of Physics, \\ The University of Sydney, New South Wales, 2006 Australia \\ ${ }^{c}$ Institute of Theoretical Physics, Chinese Academy of Sciences, \\ Beijing, 100190 China \\ ${ }^{d}$ School of Physical Sciences, University of Chinese Academy of Sciences, \\ Beijing, 100049 China \\ e Department of Physics, University of California, \\ Davis, CA, 95616 U.S.A. \\ E-mail: ghduan@itp.ac.cn, leiwu@itp.ac.cn, ruizh@ucdavis.edu
}

ABSTRACT: Searching for top squark (stop) is a crucial task of the LHC. When the flavor conserving two body decays of the stop are kinematically forbidden, the stops produced near the threshold will live long enough to form bound states which subsequently decay through annihilation into the Standard Model (SM) final states. In the region of stop mixing angle $\theta_{\tilde{t}} \rightarrow 0$ or $\pi / 2$, we note that the LHC-13 TeV diphoton resonance data can give a strong bound on the spin-0 stoponium $\left(\eta_{\tilde{t}}\right)$ and exclude the constituent stop mass $m_{\tilde{t}}$ up to about $290 \mathrm{GeV}$. While in the large stop mixing region, the stoponium will dominantly decay to the Higgs pair. By analyzing the process $p p \rightarrow \eta_{\tilde{t}} \rightarrow h(\rightarrow b \bar{b}) h\left(\rightarrow \tau^{+} \tau^{-}\right)$, we find that a large portion of the parameter space on the $m_{\tilde{t}_{1}}-\theta_{\tilde{t}}$ plane can be probed at $2 \sigma$ significance level at the LHC with the luminosity $\mathcal{L}=3000 \mathrm{fb}^{-1}$.

KeYwords: Supersymmetry Phenomenology

ArXiv EPrint: 1706.07562 


\section{Contents}

1 Introduction 1

2 Diphoton resonance constraint on the stoponium 2

3 Di-Higgs decay of stoponium with $b \bar{b} \tau^{+} \tau^{-}$final states at the LHC $\quad 4$

4 Conclusions $\quad 8$

\section{Introduction}

Since the discovery of the Higgs boson at the Run 1 of the Large Hadron Collider (LHC) in 2012 [1, 2], the persuit of physics beyond the SM (BSM) becomes the primary goal in particle physics community. One of the most important guidelines in this endeavor is the famous naturalness principle which states that the physics at weak scale should be insensitive to quantum effects from much higher scales. Among all the proposed scenarios, supersymmetry (SUSY) remains as one of the most popular models, in which the quantum correction to the Higgs mass from the top quark is canceled by that from the stop. In this regard, the search for stop [3-16] is an important direction of testing SUSY naturalness at the LHC.

Till now, numerous efforts have been dedicated to the searching for stop in the LHC experiments. The experimental signatures of stop pair production depend on the stop-LSP mass splitting which leads to different decay modes. For instance, when $m_{\tilde{t}_{1}}>m_{t}+m_{\tilde{\chi}_{1}^{0}}$ and $\tilde{t}_{1}$ mainly decays to $t \tilde{\chi}_{1}^{0}$, the top quark from stop decay can be quite energetic and a stop mass up to $940 \mathrm{GeV}$ for a massless lightest neutralino has been excluded by the very recent LHC run-2 data [17]. When the flavor-conserving two body decays channels like $\tilde{t}_{1} \rightarrow t \tilde{\chi}_{1}^{0}$ and $\tilde{t}_{1} \rightarrow b \tilde{\chi}_{1}^{+}$are kinematically forbidden, the primary decay channels of the light stop would be the three-body decay $\tilde{t}_{1} \rightarrow W^{+} b \tilde{\chi}_{1}^{0}$, the two-body flavor-changing decay $\tilde{t}_{1} \rightarrow c \tilde{\chi}_{1}^{0}$ or the four-body decay $\tilde{t}_{1} \rightarrow b f^{\prime} \bar{f} \tilde{\chi}_{1}^{0}$ [18-25]. The current null results of LHC searches for these decay channels have correspondingly excluded the stop mass up to $\sim 500 \mathrm{GeV}, 310 \mathrm{GeV}$ and $370 \mathrm{GeV}$ for certain mass splitting between the stop and the LSP [17].

It should be mentioned that such a light stop usually has very small decay width [26] compared to the typical binding energy of $\tilde{t}_{1} \tilde{t}_{1}^{*}$ bound state (stoponium). In this case, two stops produced near-threshold could live long enough to form a stoponium due to the Coulomb-like attraction via the QCD interaction. In contrast to the existing direct stop pair searches, stoponium if formed, will resonantly decay to a pair of the SM particles and can be independent of the assumptions of the LSP mass and the branching ratios of the 
stop. Therefore, it is expected that the search of stoponium can provide a complementary probe to the direct stop pair production at the LHC.

The phenomenologies of the stoponium have been studied at colliders [26-34]. In particular, the diphoton channel was studied and found to be a promising way to observe stoponium at the LHC in refs. [26-28]. The diboson decay of stoponium with $W W$ and $Z Z$ final states were also examined in $[32,33]$. In [35], the authors investigated the di-Higgs decay of stoponium with $b \bar{b} \gamma \gamma$ final states and found it to be a viable channel at the LHC. But the loop induced diphoton decay of the Higgs boson can be sizably affected by other sparticles, such as the light stau in the MSSM [36].

In this paper, we first confront the stoponium with the recent data of searching for high mass resonances at $13 \mathrm{TeV}$ LHC. Then we explore the potential of probing the stop in Higgs pair production with $b \bar{b} \tau^{+} \tau^{-}$final states at high-luminosity LHC (HL-LHC). As a comparison with $b \bar{b} \gamma \gamma$ channel, although the $b \bar{b} \tau^{+} \tau^{-}$channel suffers from relatively complicated backgrounds, it has a larger branching ratio. Besides, it is expected that the reconstruction efficiency of $\tau$ can reach $\sim 80 \%$ with the likelihood $\tau$ taggers in the future LHC experiment [37, 38]. This will make $b \bar{b} \tau^{+} \tau^{-}$channel become another promising way of discovering, or confirming the stoponium at the LHC. The paper is organized as follows. In section 2, we introduce productions and decays of the stoponium and display the limits on stoponium mass from the LHC-13 TeV data. In section 3, we investigate the observability of the di-Higgs decay of the stoponium with $b \bar{b} \tau^{-} \tau^{+}$final states at the LHC. Finally, we draw our conclusions in section 4 .

\section{Diphoton resonance constraint on the stoponium}

In the gauge-eigenstate basis, the stop mass matrix is given by

$$
M_{\tilde{t}}^{2}=\left(\begin{array}{cc}
m_{\tilde{t}_{L}}^{2} & m_{t} X_{t}^{\dagger} \\
m_{t} X_{t} & m_{\tilde{t}_{R}}^{2}
\end{array}\right)
$$

with

$$
\begin{aligned}
m_{\tilde{t}_{L}}^{2} & =m_{\tilde{Q}_{3 L}}^{2}+m_{t}^{2}+m_{Z}^{2}\left(\frac{1}{2}-\frac{2}{3} \sin ^{2} \theta_{W}\right) \cos 2 \beta, \\
m_{\tilde{t}_{R}}^{2} & =m_{\tilde{U}_{3 R}}^{2}+m_{t}^{2}+\frac{2}{3} m_{Z}^{2} \sin ^{2} \theta_{W} \cos 2 \beta, \\
X_{t} & =A_{t}-\mu \cot \beta,
\end{aligned}
$$

where $m_{\tilde{Q}_{3 L}}$ and $m_{\tilde{U}_{3 R}}$ denote the soft-breaking mass parameters of the third generation left-handed squark doublet $\tilde{Q}_{3 L}$ and the right-handed stop $\tilde{U}_{3 R}$, respectively. $A_{t}$ is the soft-breaking trilinear parameter. We neglect the generation mixing in our study. The hermitian matrix eq. (2.1) can be diagonalized by a unitary transformation:

$$
\left(\begin{array}{c}
\tilde{t}_{1} \\
\tilde{t}_{2}
\end{array}\right)=\left(\begin{array}{cc}
\cos \theta_{\tilde{t}} & \sin \theta_{\tilde{t}} \\
-\sin \theta_{\tilde{t}} & \cos \theta_{\tilde{t}}
\end{array}\right)\left(\begin{array}{c}
\tilde{t}_{L} \\
\tilde{t}_{R}
\end{array}\right),
$$


where $\theta_{\tilde{t}} \in[0, \pi)$ is the mixing angle between left-handed $\left(\tilde{t}_{L}\right)$ and right-handed $\left(\tilde{t}_{R}\right)$ stops. A very narrow decay width of stop ${ }^{1}$ can naturally appear in the compressed region, in which the decay width of stop is suppressed either by phase space or loop factor. If the $\Gamma_{\tilde{t}_{1}}$ is much smaller than binding energy, stop pair produced near the threshold could form a bound state due to the strong attractive force mediated by gluons. Then, these bound states will proceed annihilation decay rather than the prompt decay of the constituent stop.

The production of stoponium is mainly from the gluon fusion at the LHC. In narrowwidth approximation, the leading order (LO) cross section of stoponium is be given by [26]

$$
\sigma\left(g g \rightarrow \eta_{\tilde{t}}\right)=\frac{\pi^{2}}{8 m_{\eta_{\tilde{t}}}^{3}} \Gamma_{\eta_{\tilde{t}} \rightarrow g g} \frac{\hat{s}}{s} \int_{\frac{\hat{s}}{s}}^{1} \frac{d x}{x} f_{g}(x) f_{g}\left(\frac{\hat{s}}{x s}\right)
$$

where $\hat{s}$ is squared center-of-mass energy at the parton level and is taken as $\hat{s}=m_{\eta_{\tilde{t}}}^{2}$ in our calculation. $\Gamma_{\tilde{\eta}_{\tilde{t}} \rightarrow g g}$ is the width of stoponium decay to di-gluon. The next-to-leading order QCD radiative corrections to stoponium production have been calculated in [40]. We include these effects by using the values of $K$-factor given in [41].

It should be noted that there are two main uncertainties in the computation of stoponium production rate. One of them lies in the parametrization of the wavefunction, which depends on the choice of QCD scale parameter $\Lambda$ [42]. Larger value of $\Lambda$ leads to greater coupling and hence stronger binding between the constituent stops. We adopt $\Lambda=300 \mathrm{MeV}$ by following [41]. The other uncertainty comes from the contributions of excited bound states, such as $n S(n \geq 2)$ and $1 P$ states. In particular, the effects of higher $S$-wave states are compared in [41]. The excited states can contribute by either first decaying into the lowest stoponium state $(1 S)$ or decaying directly into SM final states. For instance, the non-annihilation decay of the $2 S$ state could go entirely to the $1 S$ state and the signal could be merged with that of the ground state due to the detector energy resolution [26]. In general, states with different angular momentum could have very distinct decay modes. Without thorough knowledge of the decay modes, we will take a conservative approach and focus on the $1 S$ state.

The main decay channels of the stoponium include $\eta_{\tilde{t}} \rightarrow \gamma \gamma, \gamma Z, Z Z, W W, g g, h h, t \bar{t}$. The LO partial decay widths into transverse gauge bosons are [26]

$$
\Gamma\left(\eta_{\tilde{t}} \rightarrow g g\right) \simeq \frac{4}{3} \alpha_{S}^{2} \frac{|R(0)|^{2}}{m_{\eta_{\tilde{t}}}^{2}}, \quad \Gamma\left(\eta_{\tilde{t}} \rightarrow \gamma \gamma\right) \simeq \frac{32}{27} \alpha^{2} \frac{|R(0)|^{2}}{m_{\eta_{\tilde{t}}}^{2}}
$$

where $R(0)=\sqrt{4 \pi} \psi(0)$ is the radial wavefunction at the origin. In the nonrelativistic limit $(v \rightarrow 0)$, only four-point interaction contributes to the stoponium decays $\eta_{\tilde{t}} \rightarrow g g, \gamma \gamma$. All other decay widths can be found in $[27,33]$. Radiative corrections to stoponium annihilation decays to hadrons, photons, and Higgs bosons were calculated in ref. [43].

In figure 1 , we display the decay branching ratios of the stoponium with respect to the mixing angle $\theta_{\tilde{t}}$, where we assume $\tan \beta=10, m_{\tilde{t}_{1}}=0.2 \mathrm{TeV}$ and $m_{\tilde{t}_{2}}=2 \mathrm{TeV}$. It can be seen that the stoponium dominantly decays to di-gluon when the mixing angle $\theta_{\tilde{t}}$ approaches 0 or $\pi / 2$. While if $\tilde{t}_{L}$ and $\tilde{t}_{R}$ have a sizable mixing, the stoponium will dominantly

\footnotetext{
${ }^{1}$ If the stop has a large decay width, it could in general produce a wide resonance signal and will be hardly observed on top of the continuum background [39].
} 


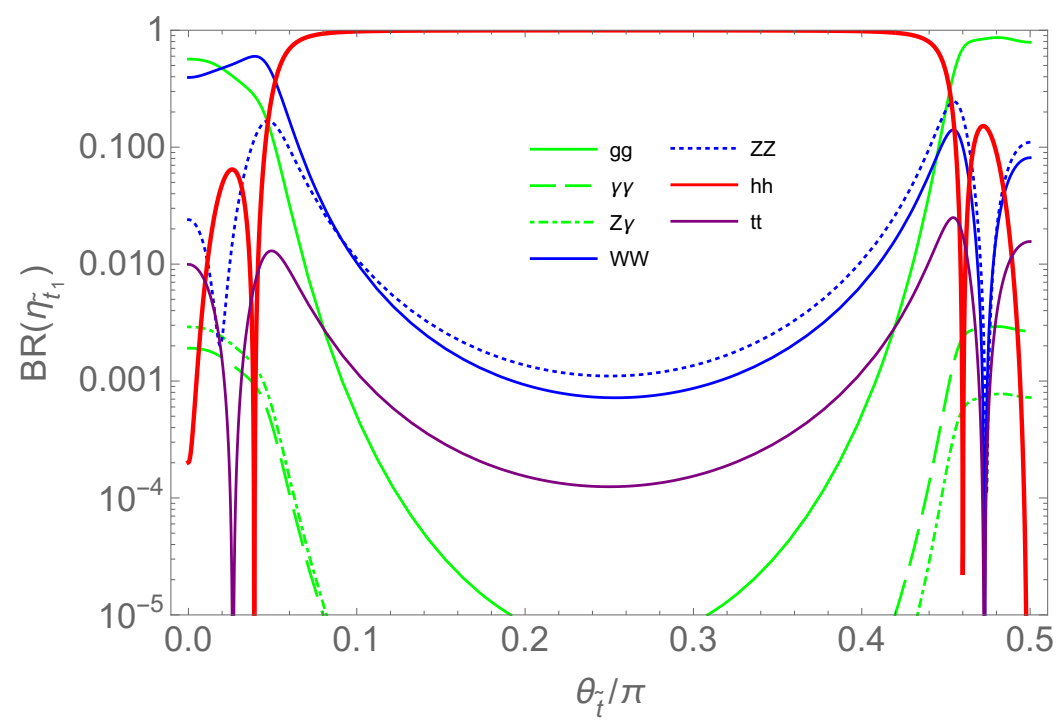

Figure 1. The decay branching ratios of the stoponium with respect to the mixing angle $\theta_{\tilde{t}}$. Here we take $\tan \beta=10, m_{\tilde{t}_{1}}=0.2 \mathrm{TeV}$ and $m_{\tilde{t}_{2}}=2 \mathrm{TeV}$ for example. Note that the branching ratios are symmetric about $\theta_{\tilde{t}}=\pi / 2$, we plot only the region $\theta_{\tilde{t}} \in[0, \pi / 2]$ here and also in figure 5 .

decay to a pair of Higgs bosons because of the enhancement induced by the Higgs-stop coupling $\lambda_{h \tilde{t}_{1} \tilde{t}_{1}}{ }^{2}$ We also checked and found that branching ratios of the stoponium have a weak dependence of $\tan \beta$. So we will assume $\tan \beta=10$ in our following calculations. Due to the distinctive signature of two photon final states, the stoponium decay to diphoton offers a very sensitive way to observing stoponium at hadron colliders.

The bound on stoponium from $8 \mathrm{TeV}$ run at the LHC is given in [44]. In figure 2, we update the result with the LHC-13 TeV diphoton resonance data [45]. We can see that the stoponium mass can be excluded up to about $580 \mathrm{GeV}$ for the mixing angles $\theta_{\tilde{t}}=\pi / 2$, which is stronger than that from LHC-13 TeV direct searches for the four-body decay $\tilde{t}_{1} \rightarrow b f^{\prime} \bar{f} \tilde{\chi}_{1}^{0}$ with pure bino LSP in the region of $m_{\tilde{t}_{1}}-m_{\tilde{\chi}_{1}^{0}}<15 \mathrm{GeV}$ [17]. However, due to the branching ratio suppression effect, there is still no constraint on the stoponium from the diphoton data for the mixing angles $\theta_{\tilde{t}}=\pi / 8, \pi / 4$. We also checked the bounds on the stoponium from current null results of LHC searches for $Z \gamma$ and diboson resonances and found that they can not give stronger limits than the diphoton data.

\section{Di-Higgs decay of stoponium with $b \bar{b} \tau^{+} \tau^{-}$final states at the LHC}

Given that the stoponium can have a large branching fraction into the two Higgs bosons, we will investigate its observability through the resonant Higgs pair production with $b \bar{b} \tau^{+} \tau^{-}$ final states at the $14 \mathrm{TeV}$ LHC,

$$
p p \rightarrow \eta_{\tilde{t}} \rightarrow h h \rightarrow b \bar{b} \tau^{+} \tau^{-},
$$

\footnotetext{
${ }^{2}$ The trilinear coupling between the SM Higgs and stop quark $\tilde{t}_{1}$ takes the form [44]: $\lambda_{h \tilde{t}_{1} \tilde{t}_{1}}=\sqrt{2} v$ $\cdot\left(\frac{m_{t}^{2}}{v^{2}}+\frac{m_{Z}^{2} c_{2 \beta}}{v^{2}}\left[c_{t}^{2}\left(\frac{1}{2}-\frac{2}{3} s_{W}^{2}\right)+s_{t}^{2}\left(\frac{2}{3} s_{W}^{2}\right)\right]+s_{t}^{2} c_{t}^{2} \frac{m_{\tilde{t}_{1}}^{2}-m_{\tilde{t}_{2}}^{2}}{v^{2}}\right)$.
} 


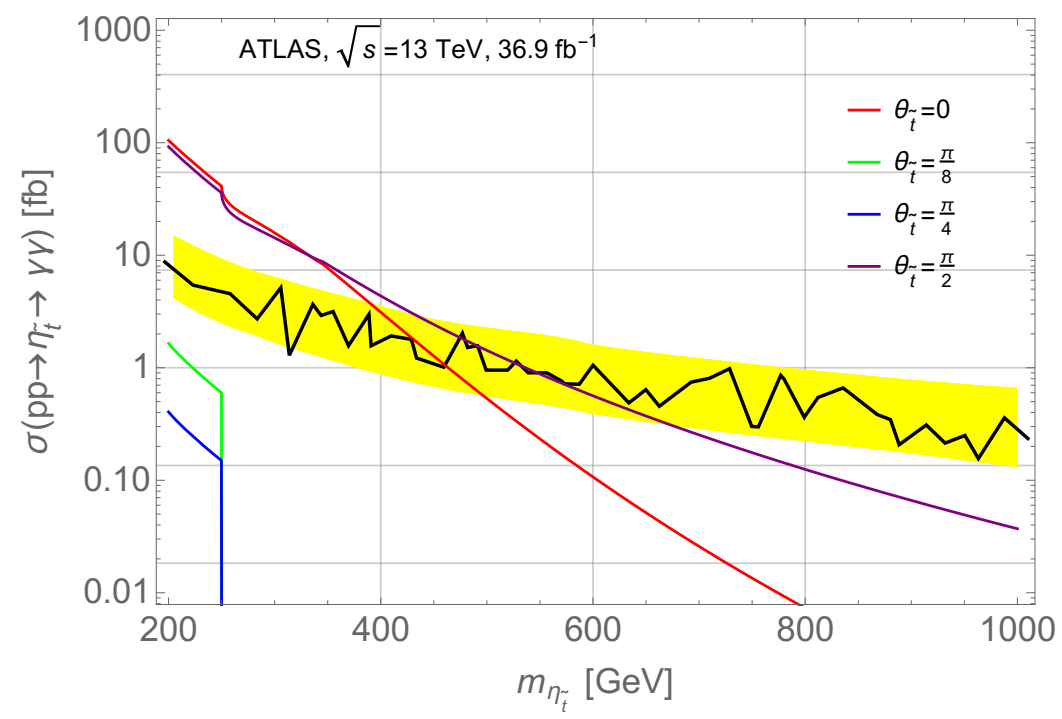

Figure 2. Constraint on the stoponium from the LHC-13 TeV diphoton resonance data. The $2 \sigma$ experimental upper limit (yellow band) is taken from [45]. Here we also assumed $\tan \beta=10$ and $m_{\tilde{t}_{2}}=2 \mathrm{TeV}$.

where one tau lepton decays hadronically $\left(\tau_{\text {had }}\right)$ and the other decays leptonically. $\tau_{\text {had }}$ is reconstructed using clusters in the electromagnetic and hadronic calorimeters with medium criterion [46].

We generate parton-level events of the stoponium production and subsequent decay into Higgs pair using the code for resonant Higgs pair production [47] within MG5_aMC@NLO [48], in which $\tau$ lepton decays are modeled by TAUOLA [49]. Then we perform parton shower and hadronization with PYTHIA [50]. The fast detector simulation is implemented with Delphes [51]. We use the $b$-jet tagging efficiency parametrization as $80 \%$ [52] and set the misidentification $10 \%$ and $1 \%$ for $c$-jets and light jets, respectively. We also assume the $\tau$ tagging efficiency is $40 \%$. We set the renormalization scale $\mu_{R}$ and factorization scale $\mu_{F}$ as the default event-by-event value. We cluster the jets by choosing the anti- $k_{t}$ algorithm with a cone radius $\Delta R=0.4$ [53]. The major backgrounds come from events with a jet misidentified as $\tau_{\text {had }}$, including $t \bar{t}, Z\left(\rightarrow \tau^{+} \tau^{-}\right) b \bar{b}$ and $Z\left(\rightarrow \tau^{+} \tau^{-}\right) j j$ processes.

In figure 3 , we present distributions of the di-tau invariant mass $m_{\tau \tau}$, two $b$-jets invariant mass $m_{b b}$, the transverse mass of the lepton plus missing energy system $m_{T}^{\ell \nu}$ and the di-tau transverse momentum $p_{T}^{\tau \tau}$. The simple transverse mass method is used to reconstruct $m_{\tau \tau}$ from the observed lepton, $\tau_{\text {had }}$ and $E_{T}^{\text {miss }}$. One can see that $m_{\tau \tau}$ distribution shows a relatively broad peak around the Higgs boson mass with a long tail, ${ }^{3}$ as a comparison with $m_{b \bar{b}}$ distribution. Another variable $m_{T}^{\ell \nu}$ can effectively reduce $t \bar{t}$ background since the lepton in signal is not from $W$ boson decay. The variable $p_{T}^{\tau \tau}$ is used to select the events with the boosted Higgs boson candidate on the transverse plane. For such events, $m_{\tau \tau}$ resolution is improved and a better separation between the signal $\eta_{\tilde{t}} \rightarrow \tau \tau$ and the

\footnotetext{
${ }^{3}$ This can be improved by using the advanced experimental MMC reconstruction technique [54].
} 

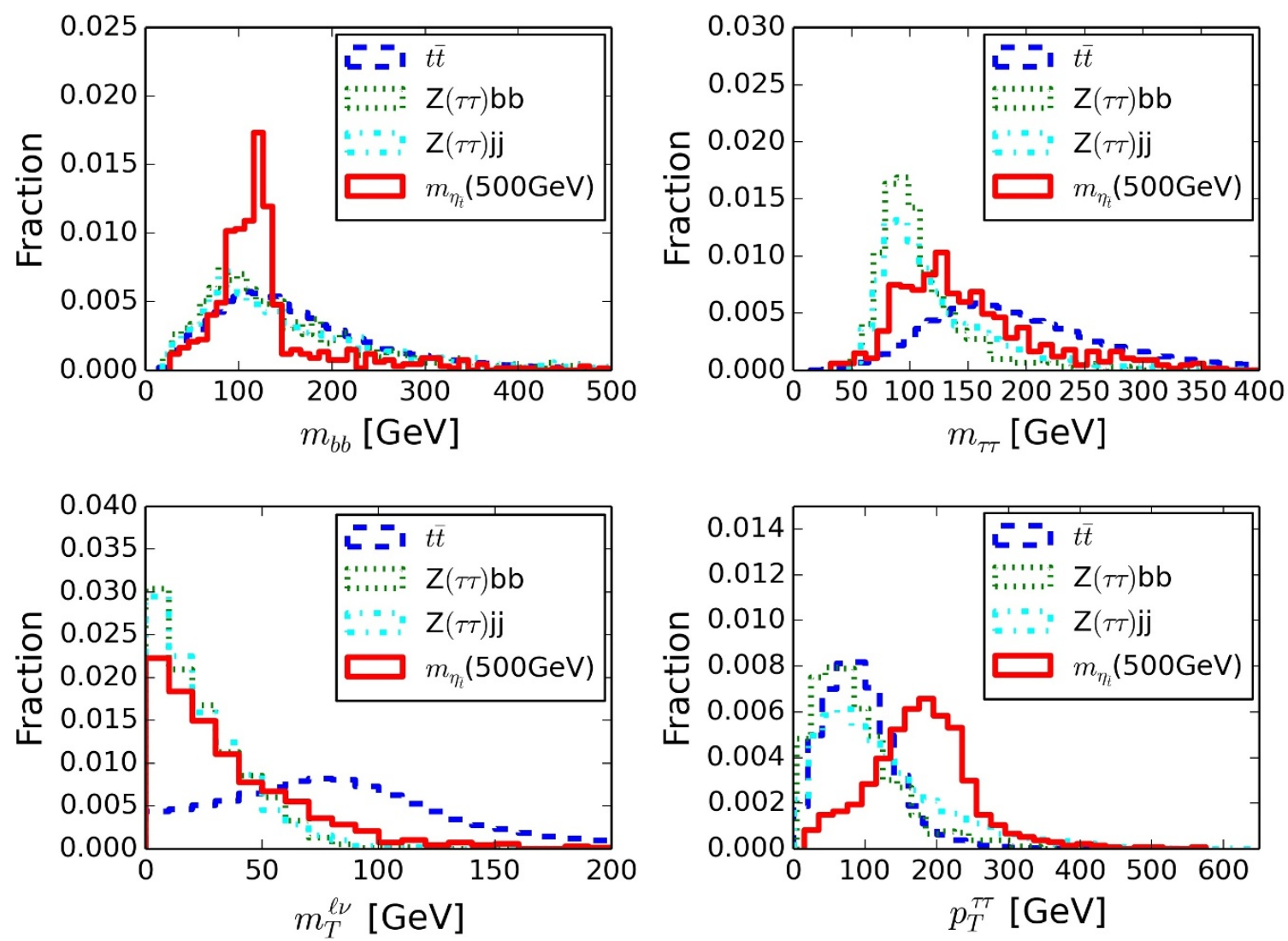

Figure 3. Distributions of the di-tau invariant mass $m_{\tau \tau}$, two $b$-jets invariant mass $m_{b b}$, the transverse mass of the lepton plus missing energy system $m_{T}^{\ell \nu}$ and the di-tau transverse momentum $p_{T}^{\tau \tau}$. The stoponium mass is taken as $m_{\eta_{\tilde{t}}}=500 \mathrm{GeV}$.

background $Z \rightarrow \tau \tau$ is achieved. This selection also has the advantage of reducing the QCD multijet background.

In our analysis, we select events that satisfy the following criteria:

- We require exactly one lepton $(e$ or $\mu)$ with $p_{T}(\ell)>26 \mathrm{GeV},\left|\eta_{e}\right|<2.47$ or $\left|\eta_{\mu}\right|<2.5$. We further require the presence of a hadronically decayed tau $\tau_{h}$ carrying opposite electric charge with $p_{T}\left(\tau_{h}\right)>20 \mathrm{GeV}$ and $\left|\eta_{\tau_{h}}\right|<2.5$.

- We require at least two jets with $p_{T}(j)>30 \mathrm{GeV}$ and $\left|\eta_{j}\right|<2.5$ and two of them are b tagged.

- We require $80 \mathrm{GeV}<m_{b b}<150 \mathrm{GeV}, 80 \mathrm{GeV}<m_{\tau \tau}<150 \mathrm{GeV}, m_{T}^{\ell \nu}<50 \mathrm{GeV}$, $p_{T}^{\tau \tau}>120 \mathrm{GeV}$ and $\left|m_{b b \tau \tau}-m_{\eta_{\tilde{t}}}\right|<0.08 m_{\eta_{\tilde{t}}}$.

In table 1, we present a cut flow of cross sections for the signal and backgrounds at $14 \mathrm{TeV}$ LHC. After the di- $b$ jets and di-tau invariant mass cuts, we find that the cut $m_{T}^{\ell \nu}<50 \mathrm{GeV}$ can reduce the $t \bar{t}$ background by about half. The cut $p_{T}^{\tau \tau}>120 \mathrm{GeV}$ can suppress $Z(\rightarrow \tau \tau) j j$ and $Z(\rightarrow \tau \tau) b b$ backgrounds by an extra factor of six. The total 


\begin{tabular}{|c|c|c|c|c|c|}
\hline Cuts & $\begin{array}{c}m_{b b} \\
\in[80,150] \mathrm{GeV}\end{array}$ & $\begin{array}{c}m_{\tau \tau} \\
\in[80,150] \mathrm{GeV}\end{array}$ & $\begin{array}{c}m_{T}^{\ell \nu} \\
<50 \mathrm{GeV}\end{array}$ & $\begin{array}{c}p_{T}^{\tau \tau} \\
>120 \mathrm{GeV}\end{array}$ & $\begin{array}{c}\left|m_{b b \tau \tau}-m_{\eta_{\bar{t}}}\right| \\
<0.08 m_{\eta_{\tilde{t}}}\end{array}$ \\
\hline$t \bar{t}$ & 445.48 & 128.79 & 55.32 & 12.46 & 0.29 \\
\hline$Z(\tau \tau) b b$ & 7.40 & 5.35 & 4.70 & 0.62 & $<0.02$ \\
\hline$Z(\tau \tau) j j$ & 11.87 & 7.92 & 7.04 & 1.62 & 0.13 \\
\hline $\operatorname{signal}\left(m_{\eta_{\bar{t}}}=500 \mathrm{GeV}\right)$ & 1.55 & 0.82 & 0.64 & 0.54 & 0.25 \\
\hline
\end{tabular}

Table 1. Cut flow analysis of the cross sections (fb) for the signal and backgrounds at $14 \mathrm{TeV}$ LHC. The benchmark point is chosen as $m_{\eta_{\tilde{t}}}=500 \mathrm{GeV}$ and $\sigma\left(g g \rightarrow \eta_{\tilde{t}} \rightarrow h h\right)=1 \mathrm{pb}$.

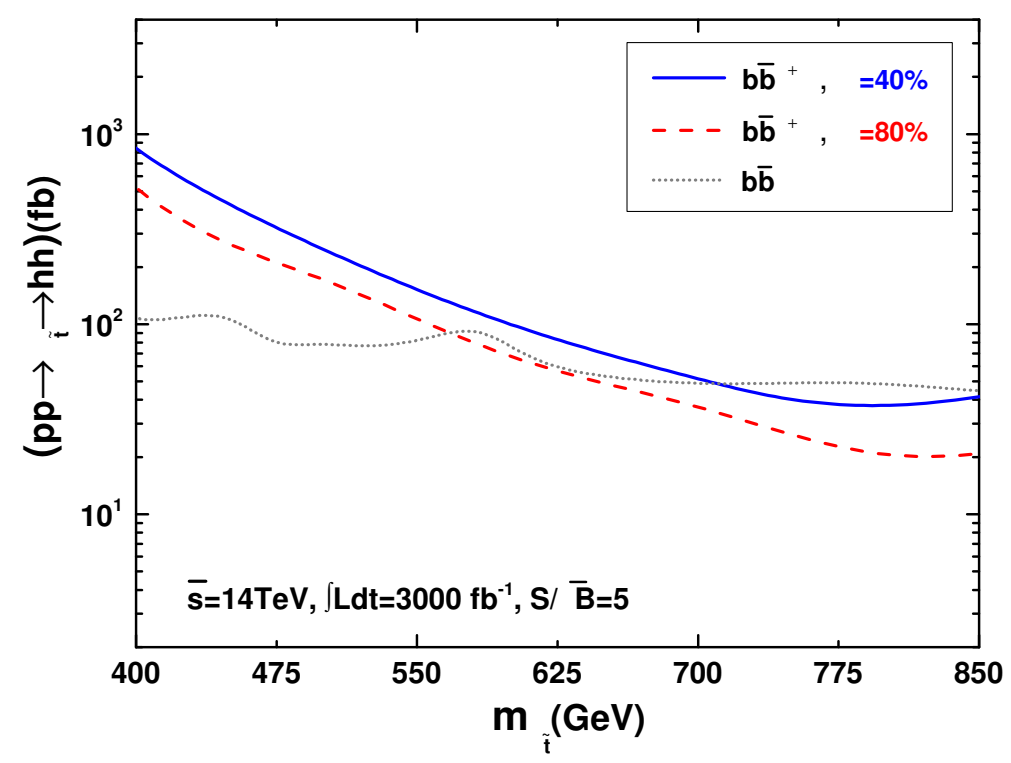

Figure 4. Cross sections of the process $p p \rightarrow \eta_{\tilde{t}} \rightarrow h h$ with $b \bar{b} \tau^{+} \tau^{-} / b \bar{b} \gamma \gamma$ final states needed for the signal significance $S / \sqrt{B}=5 \sigma$ at the HL-LHC. The result for $b \bar{b} \gamma \gamma$ final state is taken from ref. [35].

invariant mass cut $\left|m_{b b \tau \tau}-m_{\eta_{\tilde{t}}}\right|<0.08 m_{\eta_{\tilde{t}}}$ can further hurt $t \bar{t}$ background by about $\mathcal{O}\left(10^{2}\right)$ and $Z(\rightarrow \tau \tau) j j$ and $Z(\rightarrow \tau \tau) b b$ by about $\mathcal{O}(10)$.

In figure 4 , we plot the cross sections of the process $p p \rightarrow \eta_{\tilde{t}} \rightarrow h h$ with $b \bar{b} \tau^{+} \tau^{-} / b \bar{b} \gamma \gamma$ final states needed for the signal significance $S / \sqrt{B}=5 \sigma$ at the HL-LHC. It can be seen that the cross section of the process $p p \rightarrow \eta_{\tilde{t}} \rightarrow h h \rightarrow b \bar{b} \tau^{+} \tau^{-} / b \bar{b} \gamma \gamma$ should be about $800 \mathrm{fb} / 100 \mathrm{fb}$ to reach $5 \sigma$ significance at $m_{\eta_{\tilde{t}}}=400 \mathrm{GeV}$. When the stoponium is heavier than about $700 \mathrm{GeV}$, the required cross section of $b \bar{b} \tau^{+} \tau^{-}$channel for a tau tagging efficiency $\epsilon_{\tau}=40 \%$ can be comparable with that of $b \bar{b} \gamma \gamma$ channel studied in [35]. If $\tau$ tagging efficiency can be improved to $\sim 80 \%$ estimated in [37,38], the sensitivity of $b \bar{b} \tau^{+} \tau^{-}$channel is expected to become better than that of $b \bar{b} \gamma \gamma$ channel for $m_{\eta_{\tilde{t}}} \gtrsim 570 \mathrm{GeV}$.

In figure 5 , we show the $2 \sigma$ exclusion limits from the di-Higgs decay channel $\eta_{\tilde{t}} \rightarrow$ $h h \rightarrow b \bar{b} \tau^{+} \tau^{-}$and the di-photon decay channel $\eta_{\tilde{t}} \rightarrow \gamma \gamma$ for $m_{\tilde{t}_{2}}=1 \mathrm{TeV}$ and $2 \mathrm{TeV}$ on the plane of $m_{\tilde{t}_{1}}$ versus stop mixing angle $\theta_{\tilde{t}}$ at the HL-LHC. We can see that the stop mass $m_{\tilde{t}_{1}}$ can be excluded up to $\sim 380(450) \mathrm{GeV}$ in the large stop mixing region $\pi / 7 \lesssim \theta_{\tilde{t}} \lesssim \pi / 3$ 


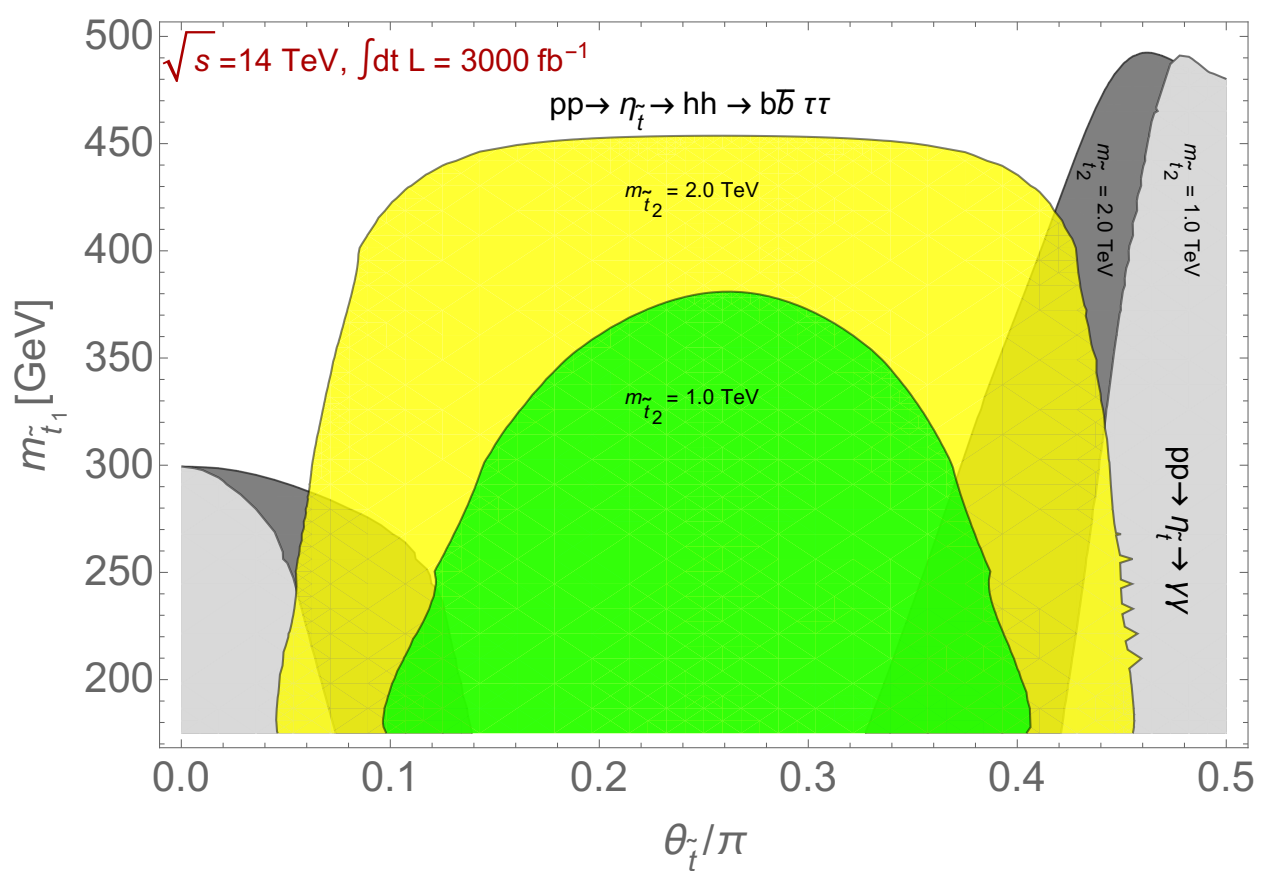

Figure 5. $2 \sigma$ exclusion limits from the di-Higgs decay channel $\eta_{\tilde{t}} \rightarrow h h \rightarrow b \bar{b} \tau^{+} \tau^{-}$and the diphoton decay channel $\eta_{\tilde{t}} \rightarrow \gamma \gamma$ for $m_{\tilde{t}_{2}}=1 \mathrm{TeV}$ and $2 \mathrm{TeV}$ on the plane of $m_{\tilde{t}_{1}}$ versus stop mixing angle $\theta_{\tilde{t}}$ at the HL-LHC. The result of di-photon decay channel is taken from ref. [44].

by the di-Higgs decay channel $\eta_{\tilde{t}} \rightarrow h h \rightarrow b \bar{b} \tau^{+} \tau^{-}$, since the branching ratio of $\eta_{\tilde{t}} \rightarrow h h$ depends on the Higgs-stop coupling $\lambda_{h \tilde{t}_{1} \tilde{t}_{1}^{*}}$. For a given mixing angle $\theta_{\tilde{t}}$, a larger $m_{\tilde{t}_{2}}$ sets a stronger bound on $m_{\tilde{t}_{1}}$ because the Higgs-stop coupling $\lambda_{h \tilde{t}_{1} \tilde{t}_{1}^{*}}$ is proportional to the mass difference $m_{\tilde{t}_{1}}^{2}-m_{\tilde{t}_{2}}^{2}$. The di-photon decay channel $\eta_{\tilde{t}} \rightarrow \gamma \gamma$ mainly excludes small stop mixing region, such as $\theta_{\tilde{t}} \lesssim \pi / 7$ or $\theta_{\tilde{t}} \gtrsim \pi / 3$, which is complementary to the di-Higgs decay channel.

\section{Conclusions}

In this paper, we confront the stoponium with the recent data of searching for high mass resonances at $13 \mathrm{TeV} \mathrm{LHC}$, and explore the potential of probing the stoponium in resonant Higgs pair production with $b \bar{b} \tau^{+} \tau^{-}$final states at the LHC. We note that the LHC-13 TeV diphoton resonance data can give a strong bound on the spin-0 stoponium $\left(\eta_{\tilde{t}}\right)$ and exclude the constituent stop mass $m_{\tilde{t}_{1}}$ up to about $290 \mathrm{GeV}$ in the small stop mixing region. While in the large stop mixing region, the stoponium will dominantly decay to Higgs pair. By analyzing the process $p p \rightarrow \eta_{\tilde{t}} \rightarrow h(\rightarrow b \bar{b}) h\left(\rightarrow \tau^{+} \tau^{-}\right)$, we find that the stop mass $m_{\tilde{t}_{1}}$ can be excluded up to $\sim 380(450) \mathrm{GeV}$ at the LHC with the luminosity $\mathcal{L}=3000 \mathrm{fb}^{-1}$.

\section{Acknowledgments}

This work was supported by the Australian Research Council, by the National Natural Science Foundation of China (NNSFC) under grants No. 11305049. 
Open Access. This article is distributed under the terms of the Creative Commons Attribution License (CC-BY 4.0), which permits any use, distribution and reproduction in any medium, provided the original author(s) and source are credited.

\section{References}

[1] ATLAS collaboration, Observation of a new particle in the search for the Standard Model Higgs boson with the ATLAS detector at the LHC, Phys. Lett. B 716 (2012) 1 [arXiv: 1207.7214] [INSPIRE].

[2] CMS collaboration, Observation of a new boson at a mass of $125 \mathrm{GeV}$ with the CMS experiment at the LHC, Phys. Lett. B 716 (2012) 30 [arXiv:1207.7235] [INSPIRE].

[3] C. Brust, A. Katz, S. Lawrence and R. Sundrum, SUSY, the Third Generation and the LHC, JHEP 03 (2012) 103 [arXiv:1110.6670] [InSPIRE].

[4] M. Papucci, J.T. Ruderman and A. Weiler, Natural SUSY Endures, JHEP 09 (2012) 035 [arXiv: 1110.6926] [INSPIRE].

[5] J. Cao, C. Han, L. Wu, J.M. Yang and Y. Zhang, Probing Natural SUSY from Stop Pair Production at the LHC, JHEP 11 (2012) 039 [arXiv:1206.3865] [INSPIRE].

[6] C. Han, K.-i. Hikasa, L. Wu, J.M. Yang and Y. Zhang, Current experimental bounds on stop mass in natural SUSY, JHEP 10 (2013) 216 [arXiv:1308.5307] [INSPIRE].

[7] A. Kobakhidze, N. Liu, L. Wu and J.M. Yang, ATLAS Z-peaked excess in the MSSM with a light sbottom or stop, Phys. Rev. D 92 (2015) 075008 [arXiv: 1504.04390] [InSPIRE].

[8] K.-i. Hikasa, J. Li, L. Wu and J.M. Yang, Single top squark production as a probe of natural supersymmetry at the LHC, Phys. Rev. D 93 (2016) 035003 [arXiv:1505.06006] [inSPIRE].

[9] M. Drees and J.S. Kim, Minimal natural supersymmetry after the LHC8, Phys. Rev. D 93 (2016) 095005 [arXiv: 1511.04461] [INSPIRE].

[10] A. Kobakhidze, N. Liu, L. Wu, J.M. Yang and M. Zhang, Closing up a light stop window in natural SUSY at LHC, Phys. Lett. B $\mathbf{7 5 5}$ (2016) 76 [arXiv:1511.02371] [INSPIRE].

[11] C. Han, J. Ren, L. Wu, J.M. Yang and M. Zhang, Top-squark in natural SUSY under current LHC run-2 data, Eur. Phys. J. C 77 (2017) 93 [arXiv:1609.02361] [INSPIRE].

[12] G.H. Duan, K.-i. Hikasa, L. Wu, J.M. Yang and M. Zhang, Leptonic mono-top from single stop production at the LHC, JHEP 03 (2017) 091 [arXiv: 1611.05211] [INSPIRE].

[13] H. Baer, V. Barger, N. Nagata and M. Savoy, Phenomenological profile of top squarks from natural supersymmetry at the LHC, Phys. Rev. D 95 (2017) 055012 [arXiv:1611.08511] [INSPIRE].

[14] M.R. Buckley, D. Feld, S. Macaluso, A. Monteux and D. Shih, Cornering Natural SUSY at LHC Run II and Beyond, arXiv:1610.08059 [INSPIRE].

[15] B. Batell, M. McCullough, D. Stolarski and C.B. Verhaaren, Putting a Stop to di-Higgs Modifications, JHEP 09 (2015) 216 [arXiv:1508.01208] [INSPIRE].

[16] C. Han, X. Ji, L. Wu, P. Wu and J.M. Yang, Higgs pair production with SUSY QCD correction: revisited under current experimental constraints, JHEP 04 (2014) 003 [arXiv: 1307.3790] [INSPIRE].

[17] ATLAS collaboration, Search for top squarks in final states with one isolated lepton, jets and missing transverse momentum using $36.1 \mathrm{fb}^{-1}$ of $\sqrt{13} \mathrm{TeV}$ pp collision data with the ATLAS detector, ATLAS-CONF-2017-037 (2017). 
[18] K.-i. Hikasa and M. Kobayashi, Light Scalar Top at $e^{+} e^{-}$Colliders, Phys. Rev. D 36 (1987) 724 [INSPIRE].

[19] T. Han, K.-i. Hikasa, J.M. Yang and X.-m. Zhang, The FCNC top squark decay as a probe of squark mixing, Phys. Rev. D 70 (2004) 055001 [hep-ph/0312129] [INSPIRE].

[20] A. Djouadi and Y. Mambrini, Three body decays of top and bottom squarks, Phys. Rev. D 63 (2001) 115005 [hep-ph/0011364] [INSPIRE].

[21] C. Boehm, A. Djouadi and Y. Mambrini, Decays of the lightest top squark, Phys. Rev. D 61 (2000) 095006 [hep-ph/9907428] [INSPIRE].

[22] R. Gröber, M.M. Mühlleitner, E. Popenda and A. Wlotzka, Light Stop Decays: Implications for LHC Searches, Eur. Phys. J. C 75 (2015) 420 [arXiv:1408.4662] [InSPIRE].

[23] M.M. Mühlleitner and E. Popenda, Light Stop Decay in the MSSM with Minimal Flavour Violation, JHEP 04 (2011) 095 [arXiv: 1102.5712] [INSPIRE].

[24] R. Gröber, M.M. Mühlleitner, E. Popenda and A. Wlotzka, Light stop decays into Wb $\tilde{\chi}_{1}^{0}$ near the kinematic threshold, Phys. Lett. B 747 (2015) 144 [arXiv: 1502. 05935] [InSPIRE].

[25] J. Aebischer, A. Crivellin and C. Greub, One-loop SQCD corrections to the decay of top squarks to charm and neutralino in the generic MSSM, Phys. Rev. D 91 (2015) 035010 [arXiv: 1410.8459] [INSPIRE].

[26] S.P. Martin, Diphoton decays of stoponium at the Large Hadron Collider, Phys. Rev. D 77 (2008) 075002 [arXiv:0801.0237] [INSPIRE].

[27] M. Drees and M.M. Nojiri, A new signal for scalar top bound state production, Phys. Rev. Lett. 72 (1994) 2324 [hep-ph/9310209] [INSPIRE].

[28] M. Drees and M.M. Nojiri, Production and decay of scalar stoponium bound states, Phys. Rev. D 49 (1994) 4595 [hep-ph/9312213] [INSPIRE].

[29] G.T. Bodwin, H.S. Chung and C.E.M. Wagner, Higgs-Stoponium Mixing Near the Stop-Antistop Threshold, Phys. Rev. D 95 (2017) 015013 [arXiv: 1609.04831] [INSPIRE].

[30] M.-x. Luo, K. Wang, T. Xu, L. Zhang and G. Zhu, Squarkonium, diquarkonium and octetonium at the LHC and their diphoton decays, Phys. Rev. D 93 (2016) 055042 [arXiv: 1512.06670] [INSPIRE].

[31] H. Ito, T. Moroi and Y. Takaesu, Di-Higgs decay of stoponium at a future photon-photon collider, Phys. Rev. D 93 (2016) 095027 [arXiv: 1602.01231] [INSPIRE].

[32] V. Barger, M. Ishida and W.-Y. Keung, Searching for Stoponium along with the Higgs boson, Phys. Rev. Lett. 108 (2012) 081804 [arXiv:1110.2147] [INSPIRE].

[33] C. Kim, A. Idilbi, T. Mehen and Y.W. Yoon, Production of Stoponium at the LHC, Phys. Rev. D 89 (2014) 075010 [arXiv:1401.1284] [InSPIRE].

[34] Z. Kang, Bound states via Higgs exchanging and heavy resonant di-Higgs, Phys. Lett. B 771 (2017) 313 [arXiv: 1606.01531] [INSPIRE].

[35] N. Kumar and S.P. Martin, LHC search for di-Higgs decays of stoponium and other scalars in events with two photons and two bottom jets, Phys. Rev. D 90 (2014) 055007 [arXiv: 1404.0996] [INSPIRE].

[36] M. Carena, S. Gori, N.R. Shah, C.E.M. Wagner and L.-T. Wang, Light Stau Phenomenology and the Higgs $\gamma \gamma$ Rate, JHEP 07 (2012) 175 [arXiv: 1205.5842] [INSPIRE].

[37] M.J. Dolan, C. Englert and M. Spannowsky, Higgs self-coupling measurements at the LHC, JHEP 10 (2012) 112 [arXiv: 1206.5001] [InSPIRE]. 
[38] M. Heldmann and D. Cavalli, An improved tau-identification for the ATLAS experiment, ATL-PHYS-PUB-2006-008 (2005) [ATL-COM-PHYS-2006-010].

[39] Y. Kats and M.D. Schwartz, Annihilation decays of bound states at the LHC, JHEP 04 (2010) 016 [arXiv: 0912.0526] [INSPIRE].

[40] K. Hagiwara, S. Matsumoto and C.S. Kim, Electroweak radiative corrections, in proceedings of the 14th International Workshop on Weak Interactions and Neutrinos (WIN 1993), Seoul, Korea, 19-24 July 1993, pp. 19-42 [hep-ph/9312231] [INSPIRE].

[41] J.E. Younkin and S.P. Martin, QCD corrections to stoponium production at hadron colliders, Phys. Rev. D 81 (2010) 055006 [arXiv:0912.4813] [InSPIRE].

[42] K. Hagiwara, K. Kato, A.D. Martin and C.K. Ng, Properties of Heavy Quarkonia and Related States, Nucl. Phys. B 344 (1990) 1 [InSPIRE].

[43] S.P. Martin and J.E. Younkin, Radiative corrections to stoponium annihilation decays, Phys. Rev. D 80 (2009) 035026 [arXiv:0901.4318] [INSPIRE].

[44] B. Batell and S. Jung, Probing Light Stops with Stoponium, JHEP 07 (2015) 061 [arXiv: 1504.01740] [INSPIRE].

[45] ATLAS collaboration, Search for new phenomena in high-mass diphoton final states using $37 \mathrm{fb}^{-1}$ of proton-proton collisions collected at $\sqrt{s}=13 \mathrm{TeV}$ with the ATLAS detector, submitted to Phys. Lett. (2017) [arXiv: 1707.04147] [INSPIRE].

[46] ATLAS collaboration, Identification and energy calibration of hadronically decaying tau leptons with the ATLAS experiment in pp collisions at $\sqrt{s}=8 \mathrm{TeV}$, Eur. Phys. J. C 75 (2015) 303 [arXiv:1412.7086] [INSPIRE].

[47] R. Frederix et al., Higgs pair production at the LHC with NLO and parton-shower effects, Phys. Lett. B 732 (2014) 142 [arXiv:1401.7340] [INSPIRE].

[48] J. Alwall et al., The automated computation of tree-level and next-to-leading order differential cross sections and their matching to parton shower simulations, JHEP 07 (2014) 079 [arXiv: 1405.0301] [INSPIRE].

[49] S. Jadach, Z. Was, R. Decker and J.H. Kuhn, The $\tau$ decay library TAUOLA: Version 2.4, Comput. Phys. Commun. 76 (1993) 361 [INSPIRE].

[50] T. Sjöstrand, S. Mrenna and P.Z. Skands, PYTHIA 6.4 Physics and Manual, JHEP 05 (2006) 026 [hep-ph/0603175] [INSPIRE].

[51] DELPHES 3 collaboration, J. de Favereau et al., DELPHES 3, A modular framework for fast simulation of a generic collider experiment, JHEP 02 (2014) 057 [arXiv:1307.6346] [INSPIRE].

[52] CMS collaboration, b-Jet Identification in the CMS Experiment, CMS-PAS-BTV-11-004 (2012).

[53] M. Cacciari, G.P. Salam and G. Soyez, The Anti-k $k_{t}$ jet clustering algorithm, JHEP 04 (2008) 063 [arXiv: 0802.1189] [INSPIRE].

[54] A. Elagin, P. Murat, A. Pranko and A. Safonov, A New Mass Reconstruction Technique for Resonances Decaying to $\tau \tau$, Nucl. Instrum. Meth. A 654 (2011) 481 [arXiv:1012.4686] [INSPIRE]. 